\title{
Novel cell death by downregulation of eEF1A1 expression in tetraploids
}

\author{
Y Kobayashi ${ }^{1}$ and S Yonehara*,1
}

When duplicated sister chromatids are not properly compacted in mitosis, chromosomes are mis-segregated, inducing genetically unstable tetraploidy known to facilitate aneuploid malignancies. Here, we show that tetraploid cells produced by impaired chromosomal condensation are eliminated by a novel type of cell death different from caspase-dependent apoptosis. The cell death was associated with downregulation of eukaryotic translation elongation factor-1 $\alpha 1$ (eEF1A1/EF-1 $\alpha$ ) expression in conjunction with accumulation of its mRNA in processing bodies ( $P$ bodies). Importantly, expression of exogenous eEF1A1 was shown to inhibit the caspase-independent cell death, and a similar cell death was observed after inducing the expression of short hairpin RNA specific for eEF1A1. Furthermore, the number of spontaneously arising binucleated cells was indicated to increase several fold during 1- to 2-week cultivation after initiation of exogenous eEF1A expression. Taken together, the novel cell death machinery should help to eliminate abnormal tetraploid cells and inhibit tumorigenesis.

Cell Death and Differentiation (2009) 16, 139-150; doi:10.1038/cdd.2008.136; published online 26 September 2008

One feature of mitosis is the condensation of chromosomes. Genetic studies using model organisms as well as RNA interference (RNAi)-mediated gene silencing analyses using human cell lines indicated that DNA topoisomerase II (topoll) and a protein complex termed condensin play a crucial role in the process of mitotic chromosomal condensation. ${ }^{1-3}$ Inhibition or depletion of these molecules induces chromosomal mis-segregation and subsequent tetraploidy. ${ }^{2-4}$ Such tetraploid errors were revealed to occur spontaneously in growing cells and facilitate tumor development. ${ }^{5-7}$ Therefore, such dangerous tetraploid cells should not progress through the cell cycle and should be eliminated in vivo. Tetraploid cells were reported to activate the 'tetraploidy checkpoint' machinery, which arrests them in the G1 phase dependent on the tumor suppressor protein $\mathrm{p} 53 ;{ }^{8}$ however, other reports recently showed that tetraploidy cannot trigger G1 arrest, ${ }^{9,10}$ and the cellular response in tetraploid cells is still unclear.

Apoptosis is known to be an important cellular mechanism inhibiting the accumulation of genomic instability, ${ }^{11}$ and caspases have a central role in the execution of apoptosis. In contrast, mounting evidence has suggested that cells can die without any associated activation of caspases. ${ }^{12,13}$ However, the molecular mechanisms and physiological roles of the caspase-independent cell death remain largely unknown.

Here, we found that a novel type of caspase-independent cell death was triggered in tetraploid cells. Cell death was executed by the downregulation of eukaryotic translation elongation factor- $1 \propto 1$ (eEF1A1, also known as EF-1 $\alpha$ ), a typical housekeeping gene product required for the maintenance of cell growth and/or survival. Moreover, the number of spontaneously arising tetraploid cells was indicated to increase in the cell death machinery-impaired cells, suggesting that the cell death machinery contributes to eliminating abnormal tetraploid cells and inhibits tumorigenesis.

\section{Results}

Activation of CREB-ER induces impaired mitotic chromosomal condensation and subsequently produces tetraploid cells. We found that abnormal activation of CAMP-responsive element-binding protein (CREB) induced failure of mitotic chromosomal condensation and subsequent tetraploidy. In a Chinese hamster ovary $(\mathrm{CHO})$ cell-derived clone stably expressing CREB-estrogen receptor (ER), a fusion protein of CREB and the mutated ligand-binding domain of estrogen receptor $\alpha$ $\left(E R^{T 2}\right),{ }^{14}$ designated as C32 (Figure 1a), cells became binucleated after treatment with a synthetic ER ligand, 4-hydroxytamoxifen (4-OHT) (Figure 1b, asterisks). CHO cells infected by a lentiviral vector encoding CREB-ER showed similar results (Supplementary Figures S1a and b), indicating that binucleation is not induced only in C32 cells

\footnotetext{
${ }^{1}$ Laboratory of Molecular and Cellular Biology, Department of Animal Development and Physiology, Graduate School of Biostudies, Kyoto University, Kyoto 606-8501, Japan

*Corresponding author: S Yonehara, Laboratory of Molecular and Cellular Biology, Graduate School of Biostudies, Kyoto University, SCRB/Building G, Yoshida Konoe-cho, Sakyo-ku, Kyoto 606-8501, Japan. Tel: + 8175753 9234; Fax: + 8175753 9235; E-mail: yonehara@lif.kyoto-u.ac.jp

Keywords: binucleated cell; caspase-independent cell death; eEF1A1; P body; tetraploid cell

Abbreviations: 4-OHT, 4-hydroxytamoxifen; 5'TOP, $5^{\prime}$ terminal oligopyrimidine tract; AIF, apoptosis-inducing factor; BrdU, 5-Bromo-2'-deoxy-uridine; CREB, cAMPresponsive element-binding protein; Dox, doxycycline; d2EGFP, destabilized enhanced green fluorescent protein; ECFP, enhanced cyan fluorescent protein; eEF1A1, eukaryotic translation elongation factor-1 $\alpha 1$; ER, estrogen receptor; EYFP, enhanced yellow fluorescent protein; FISH, fluorescence in situ hybridization; H2B, histone 2B; LDH, lactate dehydrogenase; MEF, mouse embryonic fibroblasts; P bodies, processing bodies; RNAi, RNA interference; topoll, DNA topoisomerase II; shRNA, short hairpin RNA; TetR, tetracycline repressor; UTR, untranslated region; z-VAD-fmk, benzyloxycarbonyl-Val-Ala-Asp (OMe)-fluoromethane

Received 01.7.08; revised 13.8.08; accepted 22.8.08; Edited by B Zhivotovsky; published online 26.9.08
} 


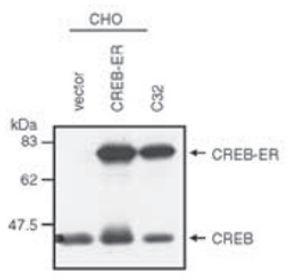

\section{b}

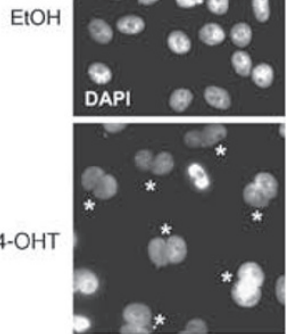

c

4-OHT

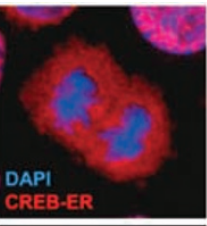

d
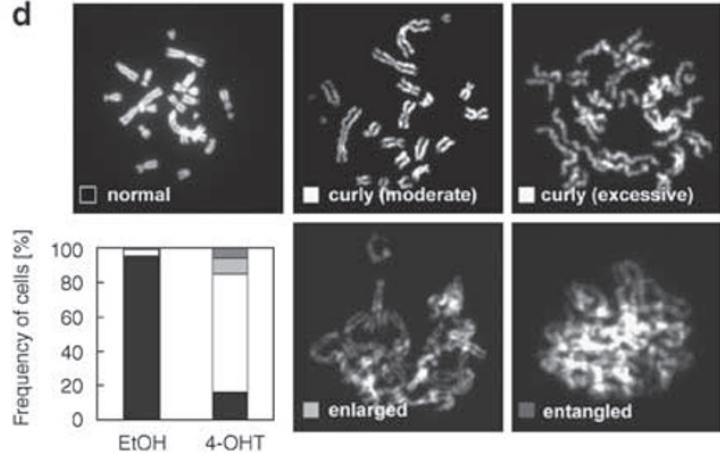

e
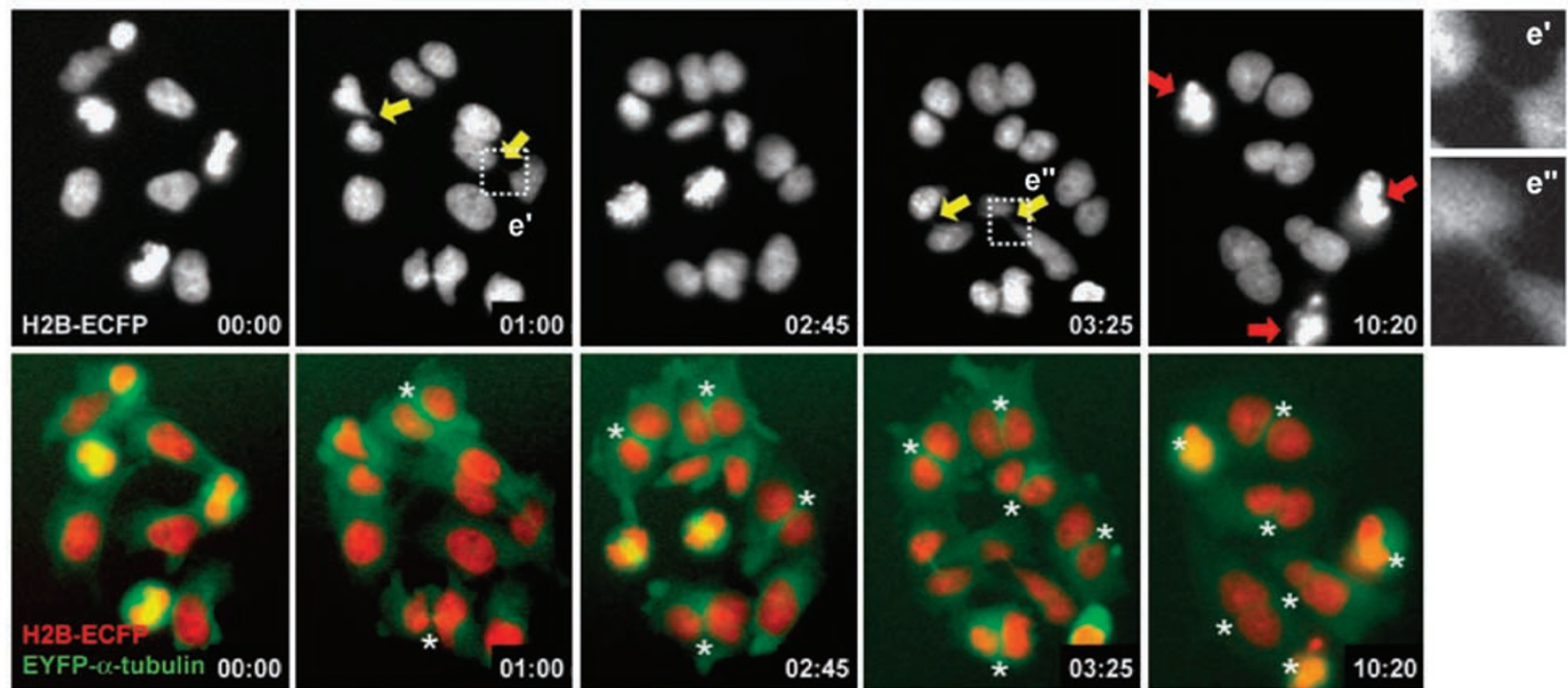

$\mathbf{f}$
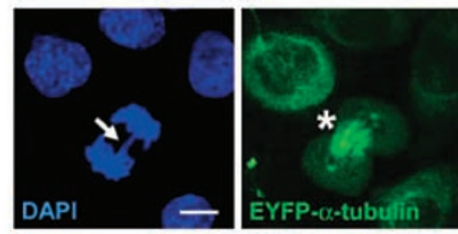

g

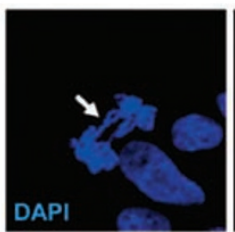

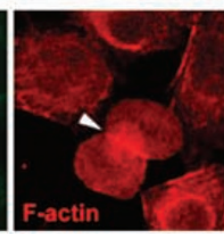

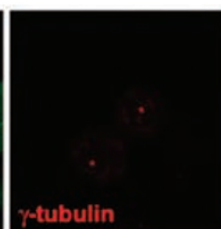

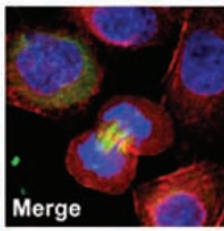

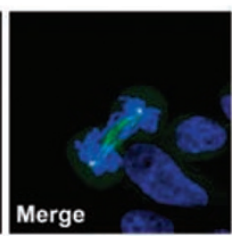

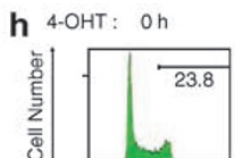
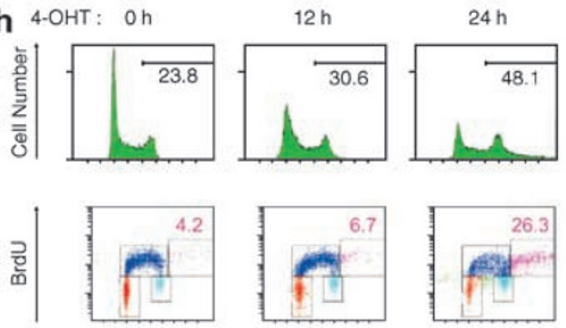

DNA content

Figure 1 Activation of CREB-ER induces impaired mitotic chromosomal condensation and subsequently produces tetraploid cells. (a) $\mathrm{C} 32$ cells and parental $\mathrm{CHO}$ cells transiently transfected with an empty vector (vector) or CREB-ER expression vector were analyzed by western blotting with anti-CREB antibody. (b) C32 cells were treated with $100 \mathrm{nM} 4-\mathrm{OHT}$ in EtOH or EtOH only for $24 \mathrm{~h}$, and DNA was stained with DAPI. Binucleated cells are indicated by asterisks. (c) C32 cells were treated with $100 \mathrm{nM} 4-\mathrm{OHT}$ in $\mathrm{EtOH}$ or EtOH only for $12 \mathrm{~h}$, and cells in anaphase were stained with anti-ER $\alpha$ antibody and analyzed under a confocal fluorescence microscope. DNA was counterstained with DAPI. (d) C32 cells were treated with $100 \mathrm{nM} 4-\mathrm{OHT}$ in EtOH or EtOH only. Metaphase chromosome spreads and percentages of cells with the different chromosome types ( $n>150$ ) are shown. (e) C32 cells stably expressing H2B-ECFP and EYFP- $\alpha$-tubulin were treated with $100 \mathrm{nM} 4-\mathrm{OHT}$ for $12 \mathrm{~h}$, and then observed under a fluorescence microscope for $12 \mathrm{~h}$. Chromosome bridges, dead cells, and binucleated cells are indicated by yellow arrows, red arrows, and asterisks, respectively. Magnified images of chromosome bridges are also shown $\left(\mathbf{e}^{\prime}\right.$ and $\left.\mathbf{e}^{\prime \prime}\right)$. (f and $\left.\mathbf{g}\right)$ After treatment with $100 \mathrm{nM} 4-\mathrm{OHT}$ for $12 \mathrm{~h}, \mathrm{C} 32$ cells stably expressing EYFP- $\alpha$-tubulin were stained with Texas Red-X phalloidin for detecting F-actin and anti- $\gamma$-tubulin antibody for detecting centrosomes, and analyzed under a confocal fluorescence microscope. DNA was counterstained with DAPI. Chromosome bridges, central spindles, and a contractile ring are indicated by arrows, asterisks, and an arrowhead, respectively. The scale bar represents $8 \mu \mathrm{m}$. (h) After treatment with $100 \mathrm{nM}$ 4-OHT, cells, pulse-labeled with $10 \mu \mathrm{M}$ BrdU for 30 min, were stained with FITC-conjugated anti-BrdU antibody and PI, and analyzed by flow cytometry. Percentages of cells in G2/M plus tetraploid cells are indicated by black. Percentages of tetraploid cells in S phase are denoted by magenta 
obtained by clonal selection. In 4-OHT-treated cells, CREBER was colocalized with chromosomes (Figure 1c and Supplementary Figure S1c) and mitotic chromosomal condensation was severely impaired (Figure 1d). The aberrant phenotypes of mitotic chromosomes in the CREBER-activated cells were also observed in cells activating M1CREB-ER, a CREB-ER mutant losing transcriptional activity $^{15}$ (Supplementary Figures S1a-C). However, activation of KCREB-ER, another CREB-ER mutant losing DNA-binding ability, ${ }^{16}$ did not provoke such phenotypes (Supplementary Figures $\mathrm{S} 1 \mathrm{a}-\mathrm{C}$ ), indicating that activation of CREB-ER induces impaired mitotic chromosomal condensation and subsequent binucleation through its DNA binding, while its transcriptional activity is dispensable. These CREB-ER-induced phenotypes (emergence of tetraploid cells with impaired mitotic chromosomal condensation) were similar to those reported earlier in cells treated with topoll inhibitors. ${ }^{4}$

To investigate the fate of cells with failure of mitotic chromosomal condensation, we generated a C32-derived cell line stably expressing histone $2 \mathrm{~B}(\mathrm{H} 2 \mathrm{~B})$-enhanced cyan fluorescent protein (ECFP) and enhanced yellow fluorescent protein (EYFP)- $\alpha$-tubulin, and continuously monitored the cells by time lapse imaging under a fluorescence microscope after treatment with 4-OHT (Figure 1e and Supplementary Movie S1). Activation of CREB-ER induced chromosome bridges in anaphase and incomplete cytokinesis, and consequently produced binucleated cells. However, the machinery of cytokinesis was shown to be intact, because C32 cells with anaphase bridges had a normal central spindle and intact contractile ring, as well as a normally oriented centrosome (Figure $1 \mathrm{f}$ and $\mathrm{g}$ ). These data suggest that cytokinesis failure was induced by abnormal chromosomes in the cleavage furrow as seen in previous reports. ${ }^{17,18}$ These binucleated cells were shown to progress through the cell cycle (Figure $1 \mathrm{~h}$ ), but finally died before entering the next $\mathrm{M}$ phase (Figure 1e and Supplementary Movie S1).

Caspase-independent cell death is induced in tetraploid cells with impaired mitotic chromosomal condensation. Then, we characterized the cell death in C32 cells. After $24-48 \mathrm{~h}$ of treatment with $4-\mathrm{OHT}$, extensive cell death was specifically induced (Figure 2a). Moreover, the cell death was little inhibited by the pan-caspase inhibitor benzyloxycarbonyl-Val-Ala-Asp (OMe)-fluoromethane (z-VADfmk) (Figure 2a). Similar results were obtained by activating CREB-ER and M1CREB-ER, but not by activating KCREB-ER, all of which were expressed by using lentiviral vectors (Supplementary Figure S1d). The caspasedependent apoptosis-inducing machinery is intact in C32 cells, because UV irradiation significantly induced the activation of caspase-3 and the fragmentation of DNA, both of which were completely inhibited in the presence of $z-V A D$ fmk (Figure $2 \mathrm{~b}$ and $\mathrm{c}$ ). In contrast, after $48 \mathrm{~h}$ of treatment with 4-OHT, only 6.9 and $11.5 \%$ of the population had an active caspase-3 and DNA fragmentation, respectively (Figure $2 \mathrm{~b}$ and c), although more than $50 \%$ of cells were found to have died (Figure 2a). These results were also confirmed by western blotting (Figure 2d). Although UV irradiation notably induced the cleavage of caspase-3 and substrates of caspases (PARP and Lamin A/C) as well as the release of cytochrome $c$ from mitochondria (Supplementary Figure $\mathrm{S} 2 \mathrm{a}), 4-\mathrm{OHT}$ treatment induced cleavage of these proteins and release of cytochrome $c$ only slightly. All these data indicate that the activation of caspase cascade was induced in an only small portion of 4-OHT-treated C32 cells and most of the dead cells were negative for the activation of caspases.

The cell death in the tetraploid cells was associated with cellular shrinkage (Supplementary Movie S1), which does not meet the criteria for necrosis associated with cellular swelling. ${ }^{13}$ Caspase-independent cell death was previously reported to be induced either by the release of apoptosisinducing factor (AIF) from mitochondria ${ }^{19}$ or by autophagy. ${ }^{20}$ In 4-OHT-treated cells, AIF was not released (Supplementary Figure S1a). Moreover, autophagosomes did not appear in 4-OHT-treated cells, and the cell death was not prevented by wortmannin, an inhibitor of autophagy, indicating that it is induced without the help of autophagic mechanisms (Supplementary Figures S2b-d).

Next, we examined whether a similar type of cell death in tetraploid cells is induced by a specific topoll inhibitor, ICRF $-193^{4}$ by using several cell lines. After treatment with ICRF-193, cell death was induced not only in C32 cells but also Balb3T3 cells and $p 53^{-1-}$ mouse embryonic fibroblasts (MEF) even in the presence of z-VAD-fmk (Figure 2e), suggesting that the cell death is executed with the help of neither p53 nor caspase activation. Although ICRF-193treated cells began to die later than CREB-ER-activated cells, this delay might be caused by ICRF-193-mediated cell cycle arrest at the G2 phase. ${ }^{21}$ However, ICRF-193-treated cells could escape the cell cycle arrest and eventually became tetraploids (data not shown) as reported earlier in various cell types. $^{4,22,23}$ The same kind of caspase-independent cell death was also observed in cells treated with another topoll inhibitor, ICRF-154 ${ }^{4}$ (Figure 2f). Taken together, a novel type of caspase-independent cell death is shown to be generally induced in tetraploid cells with impaired mitotic chromosomal condensation.

Caspase-independent cell death is induced by downregulation of eEF1A1 expression. As ICRF-193 treatment causes not only caspase-independent cell death but also G2 arrest, application of the CREB-ER system rather than ICRF-193 treatment seemed to be suitable for identifying a specific executor of cell death in tetraploid cells. Then, a cDNA subtraction experiment was carried out to investigate the genes whose expression was changed in 4-OHT-treated C32 cells. Eventually, we identified notably downregulated clones to be eEF1A1 and eukaryotic translation initiation factor 5A (elF5A). Northern blot analyses confirmed that eEF1A1 was actually decreased whereas elF5A was not in 4-OHT-treated cells (Figure 3a). Moreover, real-time quantitative RT-PCR (qRT-PCR) and western blot analyses indicated that the amount of eEF1A1 was decreased by treatment with either 4-OHT or ICRF-193 at both mRNA and protein levels, which were not changed by UV irradiation in C32 cells (Figure $3 b-e$ ). Similar results were obtained in ICRF-193-treated Balb3T3 and p53-1- MEF cells (Figure 3d). Furthermore, treatment of 4-OHT-stimulated 
a

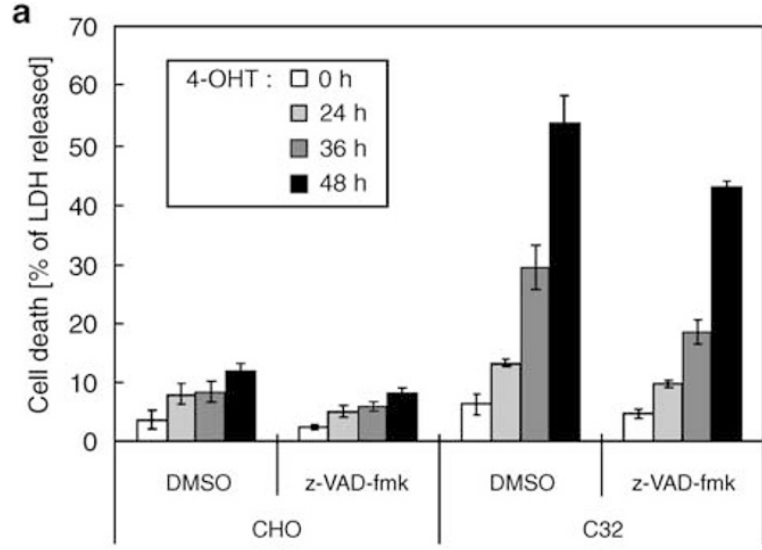

b
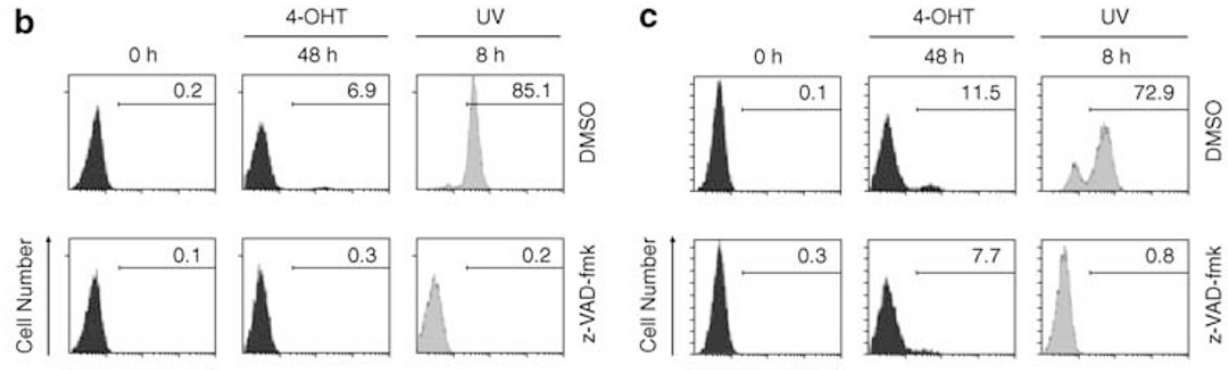

Cleaved caspase-3

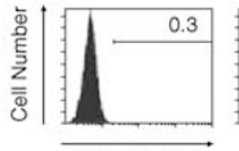

$\overline{\text { dUTP (TUNEL) }}$
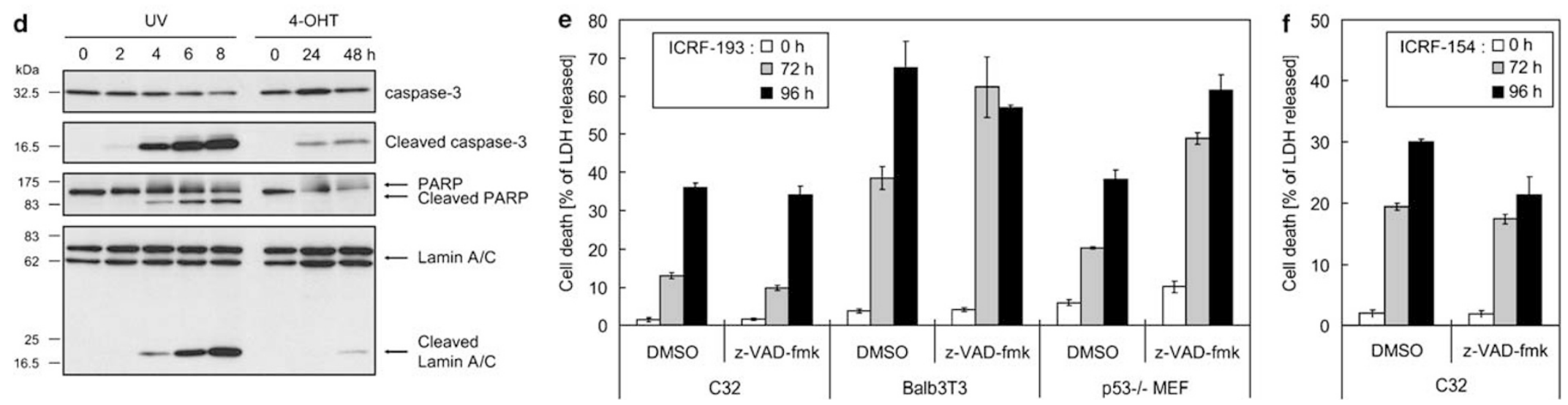

Figure 2 Caspase-independent cell death is induced in tetraploid cells. (a) C32 cells stably expressing CREB-ER and control CHO cells were treated with $100 \mathrm{nM} 4-\mathrm{OHT}$ in the presence of $50 \mu \mathrm{M} z-\mathrm{VAD}$-fmk in DMSO or DMSO only. Cell death was monitored by measuring the amount of LDH released. The data represent means \pm S.D. of a representative experiment carried out in triplicate. (b) $\mathrm{C} 32 \mathrm{cells}$ were treated with $100 \mathrm{nM} 4-\mathrm{OHT}$ or irradiated with $100 \mathrm{~mJ} / \mathrm{cm}^{2} \mathrm{UV}$, and cultured in the presence of $50 \mu \mathrm{M}$ z-VAD-fmk in DMSO or DMSO only. The amount of cleaved caspase-3 was quantified by flow cytometry after staining with anti-cleaved caspase-3 antibody. Percentages of cells with active caspase-3 are indicated in the figure. (c) After C32 cells were treated and cultured as in (b). DNA fragmentation was detected by TUNEL assay and the number of TUNEL-positive cells was quantified by flow cytometry. Percentages of TUNEL-positive cells are indicated in the figure. (d) C32 cells were treated with $100 \mathrm{nM} 4-\mathrm{OHT}$, or irradiated with $100 \mathrm{~mJ} / \mathrm{cm}^{2} \mathrm{UV}$. Cell lysates were analyzed by western blotting with anti-caspase-3, anticleaved caspase-3, anti-PARP, and anti-Lamin A/C antibodies. (e and f) Cells were treated with $10 \mu \mathrm{M}$ ICRF-193 (e) or $50 \mu \mathrm{M}$ ICRF-154 (f) in the presence of $50 \mu \mathrm{M} z-V A D$-fmk in DMSO or DMSO only. Cell death was monitored by measuring the amount of LDH released. The data represent means \pm S.D. of a representative experiment carried out in triplicate for C32 and $p 53^{-1-} \mathrm{MEF}$, and in duplicates for Balb3T3 

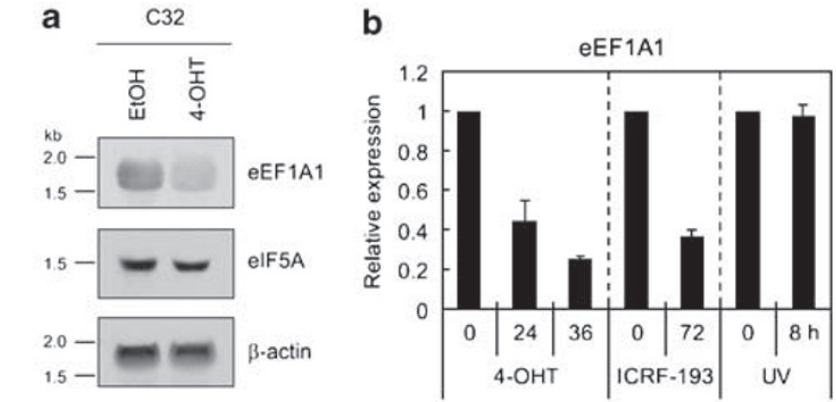

C
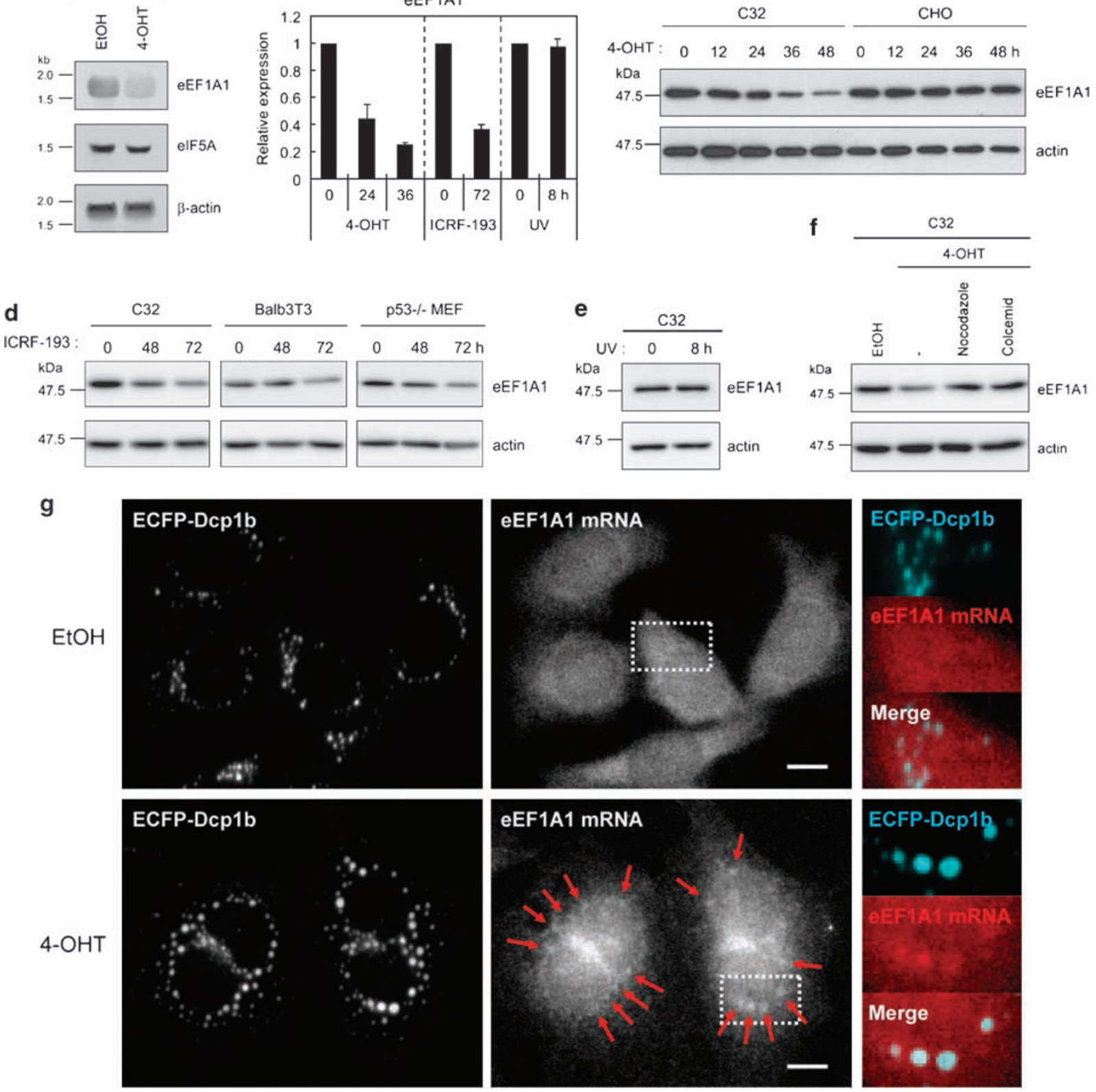

Figure 3 Expression of eEF1A1 is downregulated in tetraploid cells. (a) Northern blotting of eEF1A1, elF5A, and $\beta$-actin was carried out with total RNA extracted from C32 cells treated with $100 \mathrm{nM} 4-\mathrm{OHT}$ in EtOH or EtOH only for $30 \mathrm{~h}$. (b) C32 cells were treated with $100 \mathrm{nM} 4-\mathrm{OHT}$ or $10 \mu \mathrm{M} \mathrm{ICRF-193,} \mathrm{or} \mathrm{irradiated} \mathrm{with} 100 \mathrm{~mJ} / \mathrm{cm}^{2} \mathrm{UV}$. Real-time qRT-PCR was performed using specific primer for eEF1A1. The data represent means \pm S.D. of a representative experiment carried out in triplicate. (c-e) C32 (c and d), parental CHO (c), Balb3T3 or $p 53^{-1-}$ MEF (d) cells were treated with $100 \mathrm{nM} \mathrm{4-OHT} \mathrm{(c)} \mathrm{or} 10 \mu \mathrm{M} \mathrm{ICRF-193} \mathrm{(d),} \mathrm{or} \mathrm{irradiated} \mathrm{with} 100 \mathrm{~mJ} / \mathrm{cm}^{2} \mathrm{UV}(\mathbf{e})$, and cultured for indicated times. Cell lysates were analyzed by western blotting with anti-eEF1A and anti-actin antibodies. (f) C32 cells were treated with $0.1 \mu \mathrm{g} / \mathrm{ml}$ nocodazole or $0.1 \mu \mathrm{g} / \mathrm{ml}$ colcemid together $4-\mathrm{OHT}$ for $24 \mathrm{~h}$. Cell lysates were analyzed by western blotting with anti-eEF1A and anti-actin antibodies. (g) C32 cells stably expressing ECFP-Dcp1b were treated with $100 \mathrm{nM} 4-\mathrm{OHT}$ in EtOH or EtOH only for $24 \mathrm{~h}$, and in situ hybridization was carried out with a probe specific for eEF1A1 mRNA. eEF1A1 mRNAs colocalized with Dcp1b-positive foci (P bodies) are indicated by arrows. The scale bar represents $10 \mu \mathrm{m}$

cells with nocodazole or colcemid, both of which induce cell cycle arrest in prometaphase and inhibit the induction of binucleated cells, canceled the decreased expression of eEF1A1 (Figure 3f), indicating that downregulation of eEF1A1 is induced only after binucleation. In 4-OHT-treated cells, the eEF1A1 mRNA was accumulated in Dcp1b-positive foci, a standard marker for processing bodies ( $P$ bodies) (Figure $3 \mathrm{~g}$ ) where mRNAs are degraded or translation is repressed. ${ }^{24,25}$ These results suggest that the degradation and/or translational repression of eEF1A1 mRNA are induced in tetraploid cells.

Next, we analyzed whether the decreased expression of eEF1A1 is a direct cause of cell death. The expression of eEF1A1 was restored by infection with a lentivirus-based expression vector encoding flag-tagged eEF1A1 without its untranslated region (UTR) under the control of the $\mathrm{SR} \alpha$ 
a
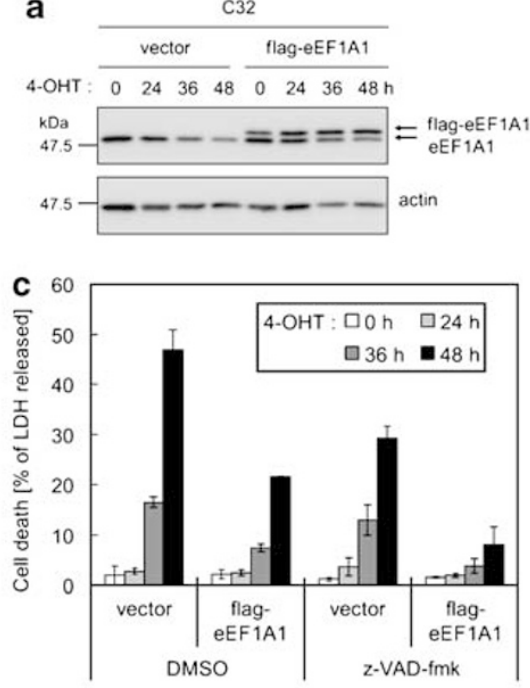

b

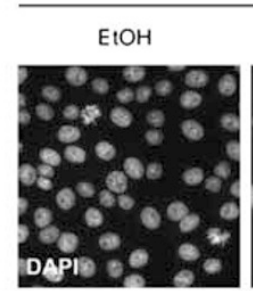

vector
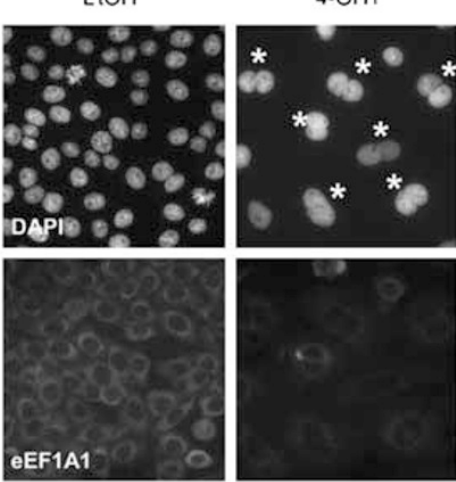

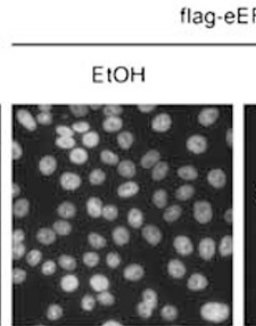

ag-eEF1A1
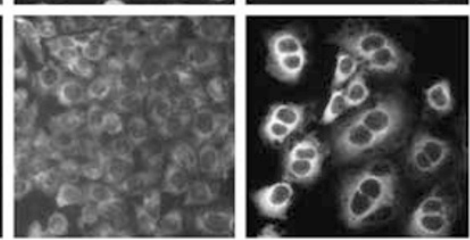

d

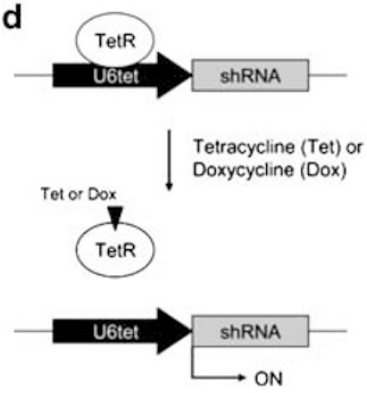

e

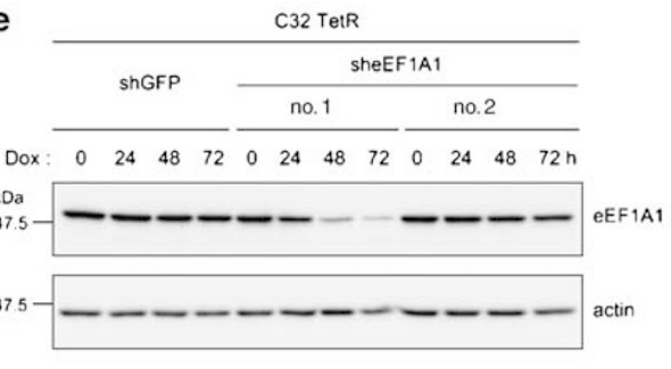

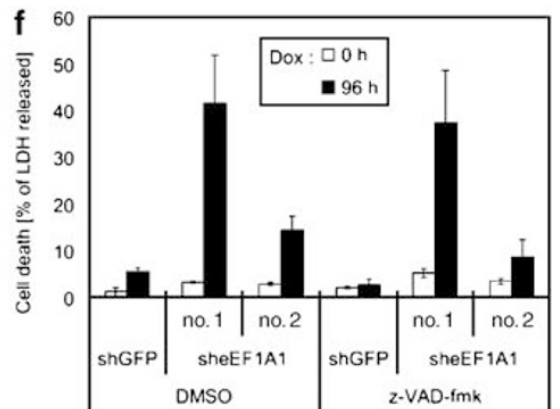

Figure 4 Caspase-independent cell death is induced by downregulation of eEF1A1 expression. (a) C32 cells infected with a lentivirus-based expression vector encoding flag-eEF1A1 or an empty viral vector (vector) were treated with $100 \mathrm{nM} 4-\mathrm{OHT}$. Cell lysates were analyzed by western blotting with anti-eEF1A and anti-actin antibodies. (b) Cells described in (a) were treated with $100 \mathrm{nM} 4-\mathrm{OHT}$, and stained with anti-eEF1A antibody. DNA was stained with DAPI. Binucleated cells are indicated by asterisks. (c) Cells described in (a) were treated with $100 \mathrm{nM} 4-\mathrm{OHT}$ in the presence of $50 \mu \mathrm{M} z-\mathrm{VAD}$-fmk in DMSO or DMSO only. Cell death was monitored by measuring the amount of $\mathrm{LDH}$ released. The data represent means \pm S.D. of a representative experiment carried out in triplicate. (d) Tetracycline-inducible shRNA expression system. (e) Tetracycline repressor (TetR)-expressing C32 cells were infected with a lentiviral vector encoding shRNA specific for GFP (shGFP) or eEF1A1 (sheEF1A1 no. 1 or no. 2). After the induction of shRNA expression by treatment with $1 \mu \mathrm{g} / \mathrm{ml}$ Dox, cell lysates were analyzed by western blotting with anti-eEF1A and anti-actin antibodies. Dox was added every $48 \mathrm{~h}$. (f) After treatment with $1 \mu \mathrm{g} / \mathrm{ml}$ Dox in the presence of $20 \mu \mathrm{M} \mathrm{z}-V A D-f m k$ in DMSO or DMSO only, cell death was quantified by measuring the amount of LDH released. Dox was added every $48 \mathrm{~h}$. The data represent means \pm S.D. of a representative experiment carried out in triplicate

promoter. We generated C32-derived cells expressing about the same amount of flag-eEF1A1 compared with endogenous eEF1A1 (Figure 4a). Flag-eEF1A1-expressing C32 cells show a similar phenotype in cell growth to empty viral vector-infected or parental cells (data not shown). The expression levels of endogenous eEF1A1 were similarly decreased in all the cells after treatment with $4-\mathrm{OHT}$, whereas the expression level of flag-eEF1A1 was not decreased whether 4-OHT was treated or not (Figure 4a). The staining of eEF1A1 in control cells showed that eEF1A1 expression was downregulated in binucleated cells (Figure $4 \mathrm{~b}$ ). Binucleation was similarly produced in the exogenous eEF1A1-expressing cells (Figure $4 \mathrm{~b}$, asterisks). In the flag-eEF1A1-expressing cells, cell death was significantly inhibited, and importantly, it was fairly canceled by treatment with z-VAD-fmk (Figure 4c), suggesting that caspase-independent cell death is induced by downregulation of eEF1A1 expression.

We further investigated whether the decreased expression of eEF1A1 itself can induce caspase-independent cell death by using a tetracycline-induced short hairpin RNA (shRNA) expression system (Figure 4d). We prepared two kinds of lentiviral vectors encoding shRNAs against eEF1A1 (sheEF1A1 no. 1 and no. 2). The induced expression of these shRNAs by treatment with doxycycline (Dox) showed that sheEF1A1 no. 1 could, whereas sheEF1A1 no. 2 could not, effectively downregulate the expression of eEF1A1 (Figure 4e). After incubation with Dox, cell death was specifically induced in cells expressing sheEF1A1 no. 1 even in the presence of z-VAD-fmk, indicating that the downregulation of eEF1A1 itself can induce caspase-independent cell death (Figure 4f). Taken together, the decreased expression of eEF1A1 is the main factor contributing to the induction of the novel type of caspase-independent cell death in tetraploid cells.

Caspase activation is induced in a small portion of the tetraploid cells in a p53-dependent manner. We also identified that a small portion of the tetraploid cells died in a caspase-dependent manner (Figure 2a). When chromosomes are bridged in mitosis, some chromosomes might 


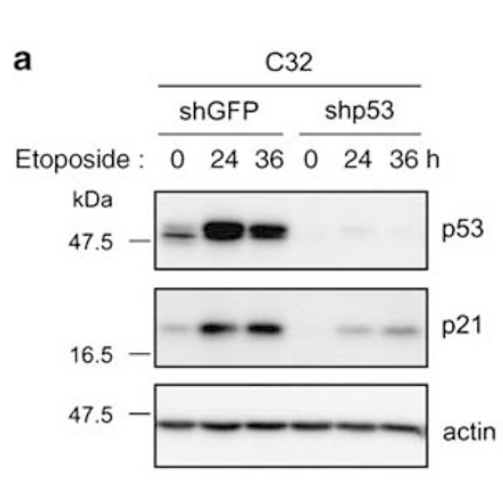

b

ShGFP $\mathrm{C} 32$

4-OHT: $\begin{array}{llllllll}0 & 24 & 36 & 48 & 0 & 24 & 36 & 48\end{array}$

$\mathrm{kDa}$
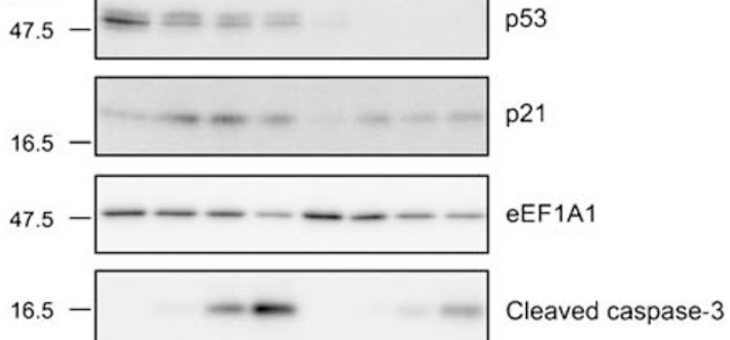

$47.5------a$ actin

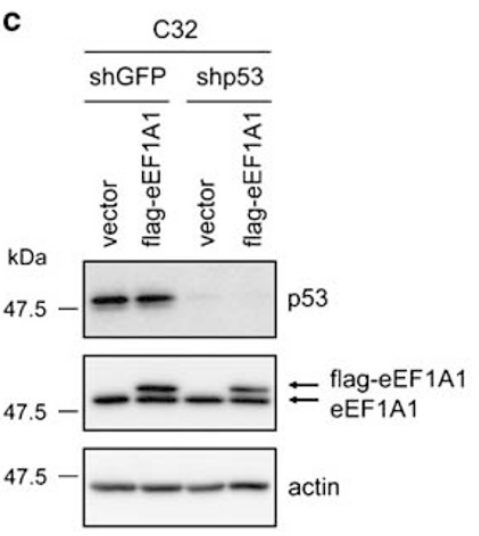

d

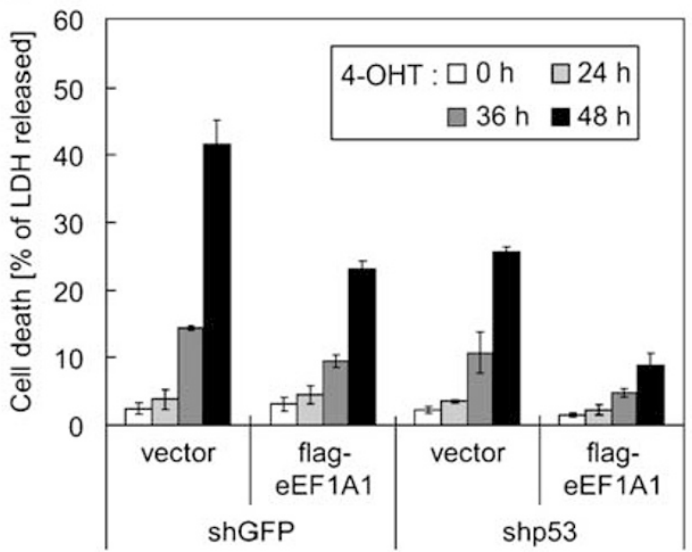

Figure 5 Caspase activation is induced in a small portion of tetraploid cells in a p53-dependent manner. (a and $\mathbf{b})$ C32 cells were infected with a lentivirus-based expression vector encoding shRNA specific for GFP (shGFP) or p53 (shp53). After the treatment with $50 \mu \mathrm{M}$ etoposide (a) or $100 \mathrm{nM} 4-\mathrm{OHT}$ (b), cell lysates were analyzed by western blotting with antibodies specific to the indicated proteins. (c) Cells described in Figure 4a were infected with a lentivirus-based expression vector encoding shGFP or shp53. Cell lysates were analyzed by western blotting with anti-p53, anti-eEF1A, and anti-actin antibodies. (d) Cells described in (c) were treated with 100 nM 4-OHT. Cell death was monitored by measuring the amount of $\mathrm{LDH}$ released. The data represent means \pm S.D. of a representative experiment carried out in triplicate

be broken when pulled to opposite spindle poles, which would trigger p53-dependent checkpoint-stimulating apoptosis. $^{26}$ To examine whether p53 is involved in the caspase-dependent cell death, expression of p53 was downregulated by the lentiviral-based expression of shRNA against p53 (shp53). Although $\mathrm{CHO}$ cells were previously reported to carry a mutation in p53 (T211K), ${ }^{27}$ expression of both p53 and p21 was upregulated in C32 cells after treatment with etoposide, a DNA damage-inducing reagent (Figure 5a). Moreover, their upregulation was diminished in shp53-expressing cells (Figure 5a), indicating that C32 cells used here provide intact p53 machinery. In contrast, expression of neither p53 nor p21 was upregulated in 4-OHT-treated C32 cells (Figure $5 \mathrm{~b}$ ). The weak activation of caspase-3 was diminished in shp53-expressing cells (Figure $5 \mathrm{~b}$ ), indicating that caspase activation is triggered in a small portion of the tetraploids in a p53-dependent manner. Importantly, downregulation of eEF1A1 expression was not affected in shp53-expressing cells (Figure 5b), and the 4-OHT-induced cell death was synergistically inhibited by the knockdown of p53 expression and exogenous expression of eEF1A1 (Figure 5c and d). These data indicate that the tetraploid cells are eliminated by either caspase-independent cell death induced by downregulation of eEF1A1 expression or p53-induced caspase-dependent apoptosis.

Expression of eEF1A1 is regulated by $5^{\prime} U T R$ region of its mRNA. As exogenously expressed eEF1A1 without the UTR was not downregulated in 4-OHT-treated cells (Figure 4a), we hypothesized that UTR is important for the downregulation of eEF1A1 expression. To examine the importance of the UTR of eEF1A1 mRNA, we generated an expression vector for destabilized enhanced green fluorescent protein (d2EGFP), having an approximately 2-hour half-life in vivo, driven by the eEF1A1 promoter (EF). In this system, transcribed d2EGFP mRNA contains the $5^{\prime}$ UTR of eEF1A1 mRNA ${ }^{28}\left(5^{\prime}\right.$-d2EGFP) (Figure 6a). The $\mathrm{SR} \alpha$ promoter was used as a control without the $5^{\prime}$ UTR (d2EGFP). We also generated expression vectors for d2EGFP with the $3^{\prime}$ UTR of eEF1A1 mRNA (5'-d2EGFP-3' and $\left.d 2 E G F P-3^{\prime}\right)$. After treatment with $4-\mathrm{OHT}$, the expression of eEF1A1 mRNA and d2EGFP mRNA with the 5'UTR was 

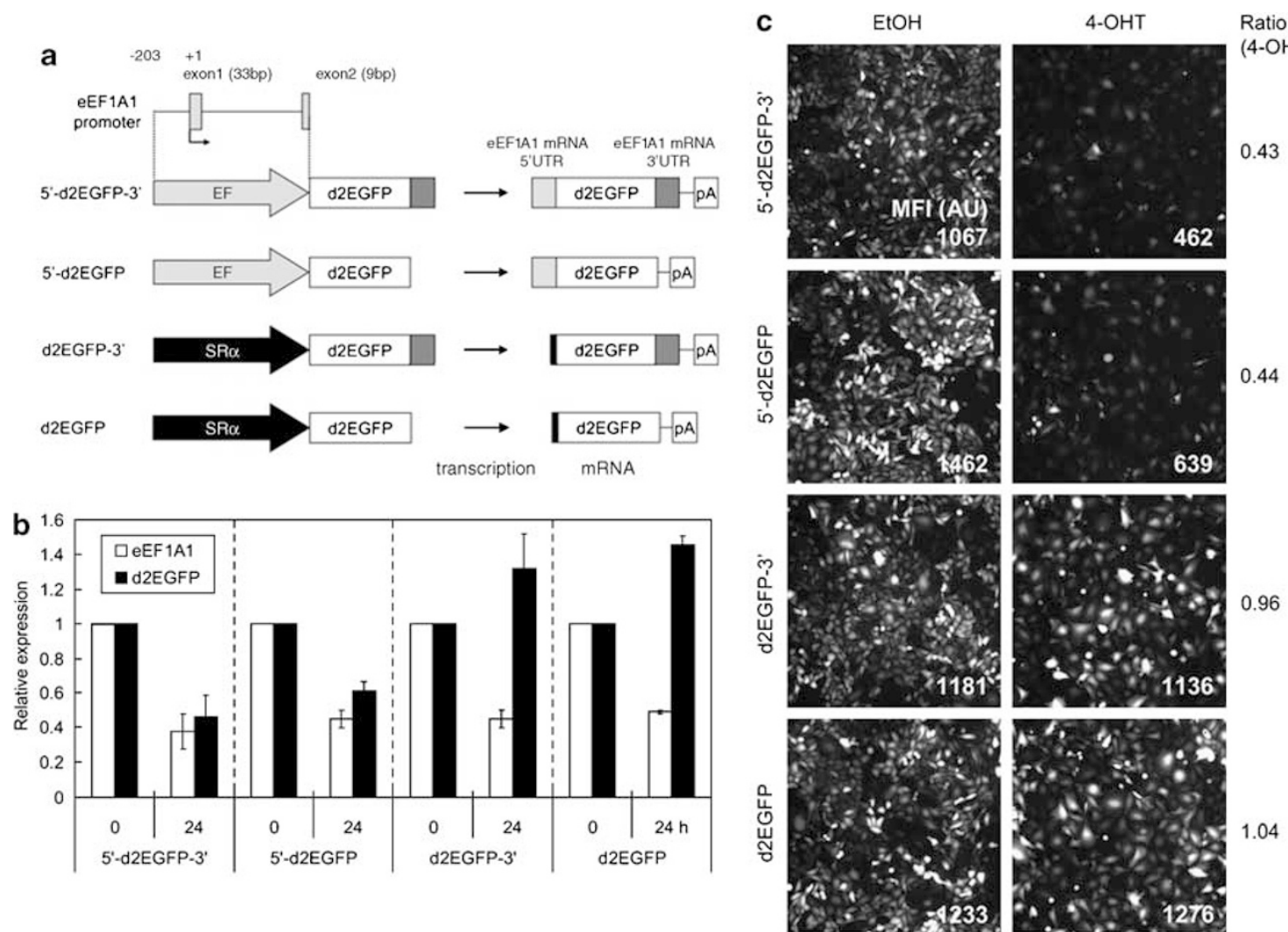

Figure 6 Expression of eEF1A1 is downregulated through the 5'UTR of its mRNA. (a) Depiction of expression vectors for d2EGFP. (b) C32 cells were infected with a lentivirus-based expression vector described in (a). After treatment with $100 \mathrm{nM}$ 4-OHT, real-time qRT-PCR was performed using specific primers for eEF1A1 or d2EGFP. The data represent means \pm S.D. of a representative experiment carried out in triplicate. (c) Cells described in (b) were treated with $100 \mathrm{nM} 4-\mathrm{OHT}$ in EtOH or EtOH only for $24 \mathrm{~h}$. The mean fluorescence intensity (MFI) in d2EGFP-expressing cells was quantified. The ratios of MFI between EtOH- (control) and 4-OHT-treated cells are indicated. AU, arbitrary units of MFI

similarly reduced, whereas that of d2EGFP mRNA without the $5^{\prime}$ UTR was not reduced (Figure $6 \mathrm{~b}$ ). These results were confirmed by analyses of the fluorescence intensity caused by expression of d2EGFP protein (Figure 6c). The $5^{\prime}$ UTR was clearly indicated to be important for the downregulation of eEF1A1 expression.

The number of spontaneously arising binucleated cells are increased in the cell death machinery-impaired cells. Binucleated tetraploid cells were reported to spontaneously arise in a small but significant population of growing cells with a failure of cytokinesis induced by chromosome nondisjunction, ${ }^{6}$ chromosome bridges in the cleavage furrow, ${ }^{17}$ and so on. Here, we showed naturally arising impaired mitotic chromosomal condensation in as many as $4.6 \%$ of normally growing cells (Figure 1c, EtOH), which is thought to induce binucleation. To investigate whether the novel type of cell death machinery is involved in eliminating spontaneously arising binucleated cells, we analyzed the frequency of spontaneous binucleation in normally growing C32 and Balb3T3 cells (Figure 7a and b). Although less than $1 \%$ of growing cells were binucleated, the number of binucleated cells was significantly increased within 1- to 2-week cultivation after initiation of exogenous eEF1A1 expression. We also analyzed the effects of exogenous expression of eEF1A2, a homolog of eEF1A1 ${ }^{29}$ In $\mathrm{C} 32$ cells, expression of endogenous eEF1A2 was undetectable (Supplementary Figure S3), and exogenous expression of eEF1A2, which inhibits the cell death in tetraploids as well as eEF1A1 (data not shown), was shown to similarly increase the number of binucleated cells (Figure 7b). From these data, the cell death machinery is suggested to contribute to eliminate naturally arising binucleated tetraploid cells.

\section{Discussion}

As tetraploid errors in growing cells cause aneuploid malignancies, ${ }^{5-7}$ tetraploid cells should not progress through the cell cycle and should be eliminated in vivo. Such dangerous cells were reported to activate the 'tetraploidy checkpoint' machinery which arrests them in the G1 phase dependent on the tumor suppressor protein $\mathrm{p} 53 .{ }^{8}$ However, the tetraploid cells examined here were shown to progress through the cell cycle without G1 arrest (Figure 1h) despite having intact p53 machinery (Figure 5a). These results are consistent with recent reports that tetraploidy cannot trigger G1 arrest in mammalian somatic cells, ${ }^{9,10}$ and other mechanisms must be activated to prevent the accumulation of abnormal cells. 
a

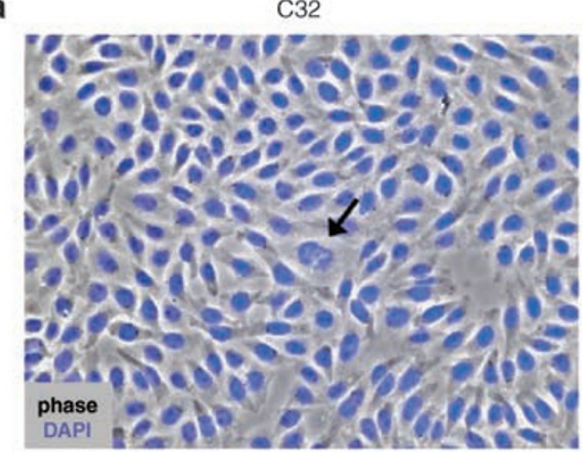

C32 flag-eEF1A1

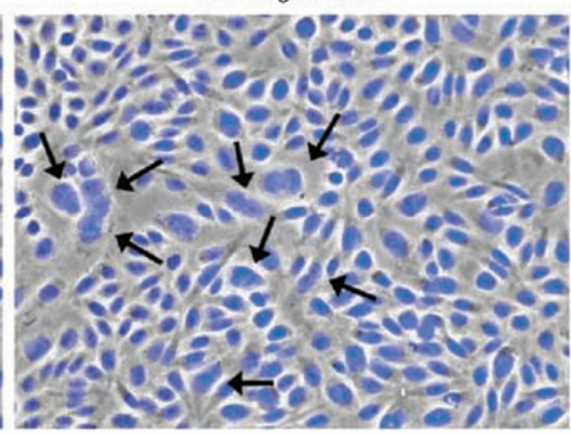

b

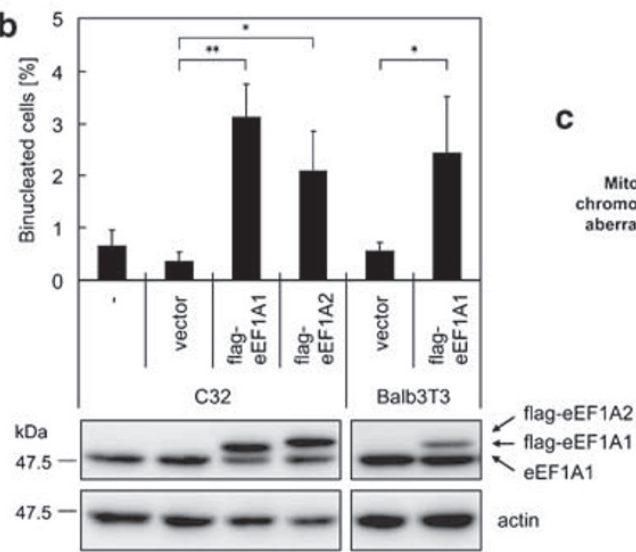

Figure 7 Number of spontaneously arising binucleated cells are increased in the cell death machinery-impaired cells. (a and $\mathbf{b})$ Cells were infected with a lentivirus-based expression vector encoding flag-eEF1A1 or flag-eEF1A2, or an empty viral vector (vector), and cultured for 1-2 weeks. C32 (Control) and flag-eEF1A1-expressing C32 cells were observed under a fluorescence microscope. DNA was stained with DAPI (a). Percentages of spontaneously arising binucleated cells were quantified $(n>457$ for $\mathrm{C} 32$, $n>356$ for Balb3T3) (b). The data represent means \pm S.D. of three independent experiments. Asterisks show significant differences by Student's $t$-test $\left({ }^{*} P<0.05\right.$; $\left.{ }^{* *} P<0.005\right)$. Exogenous expression of flag-eEF1A was confirmed by western blotting with anti-eEF1A and anti-actin antibodies. (c) Model for the novel type of cell death induced by downregulation of eEF1A1 expression in tetraploid cells

Here, we found that downregulation of eEF1A1 expression was induced in tetraploid cells (Figure 3 ), which is the main factor contributing to the induction of cell death (Figure 4). As the downregulation of eEF1A1 expression was canceled by inhibiting binucleation (Figure $3 f$ ), the downregulation was suggested to be introduced only in binucleated cells. In fact, we observed the cell death only among binucleated cells (Figure 1e and Supplementary Movie S1) progressing through the cell cycle (Figure $1 \mathrm{~h}$ ), which has been regarded to enhance genomic instability. ${ }^{5}$ We also showed that a small portion of the tetraploid cells died by p53-dependent apoptosis (Figure 5). When chromosomes are bridged in mitosis, some chromosomes would be broken, inducing apoptosis in a p53dependent manner. ${ }^{26}$ Hence, we anticipate that p53-dependent apoptosis is activated only a small portion of the tetraploid cells with physical damages in their chromosomes. In contrast, the majority of the tetraploid cells without severe DNA damages would be eliminated by downregulation of eEF1A1 expression. These two different types of cell death machinery would coordinately function to eliminate abnormal tetraploid cells.

The downregulation of eEF1A1 expression was shown to occur in conjunction with accumulation of its mRNA in $P$ bodies (Figure $3 \mathrm{~g}$ ). $\mathrm{P}$ bodies are specialized sites of $\mathrm{mRNA}$ regulation, involving in the translational repression and degradation of mRNAs. ${ }^{24,25}$ Earlier reports showed that approximately $70 \%$ of eEF1A1 mRNA is in polysomes in normally growing cells. ${ }^{30}$ In tetraploid cells, however, accumulation of eEF1A1 mRNA in P bodies was accelerated, suggesting that the redistribution of eEF1A1 mRNA from polysomes to $\mathrm{P}$ bodies causes reduced expression of eEF1A1.

eEF1A1 mRNA is classified as a $5^{\prime}$ terminal oligopyrimidine tract (5'TOP) mRNA, containing an oligopyrimidine tract immediately downstream of the transcription start site. ${ }^{31}$ An earlier report suggested that several 5'TOP mRNA-binding proteins such as La autoantigen ( $\mathrm{La}$ ) and cellular nucleic acid binding protein (CNBP) can bind to $5^{\prime}$ UTR of 5'TOP mRNAs and regulate their expression in vitro, ${ }^{32}$ it is possible that these mRNA-binding proteins have a role in the regulation of the expression of eEF1A1.

Other translation-related genes such as eEF2 and ribosomal proteins (RPs) are also known to be encoded by $5^{\prime}$ TOP mRNA. $^{31}$ Therefore it is also possible that these $5^{\prime}$ TOP mRNA-derived proteins are downregulated in tetraploid cells, synergistically inducing translational inhibition. Indeed, we observed that the expression of several 5'TOP mRNA-derived proteins was actually downregulated to some degree in tetraploid cells (data not shown). However, eEF1A1 was most prominently downregulated among the 5'TOP 
mRNA-encoded proteins we examined. Furthermore, restored expression of eEF1A1 significantly canceled the caspase-independent cell death in tetraploids (Figure 4c), indicating that the reduction in the expression of eEF1A1, rather than other $5^{\prime}$ TOP $\mathrm{mRNA}$-derived proteins, is the main factor contributing to the induction of caspase-independent cell death.

We also showed the probability that spontaneously arising binucleated cells were eliminated in normally growing cells by the novel cell death machinery. Binucleated tetraploid cells were reported to spontaneously arise with a failure of cytokinesis with chromosomal defects in mammalian cells. ${ }^{6,17}$ Although a substantial population of such binucleated cells was observed to have died, ${ }^{6}$ the molecular mechanisms have not been clarified. We showed that the number of spontaneously arising binucleated tetraploid cells was significantly increased among cells expressing exogenous eEF1A (Figure $7 \mathrm{~b}$ ). Hence, we suppose that the cell death machinery found in this study should contribute to the elimination of naturally arising tetraploid cells and inhibition of tumorigenesis.

There are two forms of eEF1A in eukaryotes, eEF1A1 and eEF1A2, both of which possess the same role in protein synthesis. $^{29}$ eEF1A2 was reported to be exclusively expressed in brain, heart, and skeletal muscle, whereas eEF1A1 is well known to be ubiquitously expressed. ${ }^{33}$ We confirmed that the expression of eEF1A2 was undetectable in cell lines used here (Supplementary Figure S3), suggesting that downregulation of eEF1A1 expression in tetraploid cells generally induces loss of eEF1A expression resulting in severe inhibition of translation. Interestingly, eEF1A2 was reported to be overexpressed in many cancer tissues, ${ }^{34,35}$ and ectopic expression of wild-type eEF1A2 enhanced tumorigenecity. ${ }^{34}$ However, it has not been clarified how overexpression of eEF1A2 contributes to tumorigenesis. As 5'UTR of eEF1A2 mRNA have no significant similarity to that of eEF1A1 mRNA, eEF1A2 was considered not to be downregulated in tetraploid cells. In addition, exogenous expression of eEF1A2 inhibits the cell death in tetraploids as well as eEF1A1 (data not shown). Hence, we anticipate that overexpression of eEF1A2 facilitates tumor development through diminishing the cell death induced by downregulation of eEF1A1.

In conclusion, we show here that downregulation of eEF1A1 expression was induced in tetraploid cells, which contributes to the elimination of tetraploid cells and inhibits tumorigenesis (Figure 7c). Our results provide not only information on novel cell cycle checkpoint machinery eliminating tetraploid cells, but also a clue for understanding the molecular mechanisms and physiological roles of caspaseindependent cell death.

\footnotetext{
Materials and Methods

Cell lines. Chinese hamster ovary cells were cultured in Ham's F12 medium (Nacalai Tesque Inc.). Mouse BalB3T3 fibroblasts, $p 53^{-1-}$ MEF, and human mammary gland adenocarcinoma MCF-7 cells were cultured in Dulbecco's modified Eagle's medium (Nacalai Tesque Inc.). $p 53^{-1-}$ MEF was provided by $O$ Niwa (Kyoto University). Cells were cultured with the media supplemented with $10 \%$ fetal bovine serum, $100 \mathrm{U} / \mathrm{ml}$ penicillin, and $100 \mu \mathrm{g} / \mathrm{ml}$ streptomycin at $37^{\circ} \mathrm{C}$ in $5 \% \mathrm{CO}_{2}$.
}

Plasmids. The expression vector for CREB was a gift from J Fujisawa (Kansai Medical Collage, Osaka). The expression vectors for ER for CREB-ER and GFP$\mathrm{LC}^{36}$ were gifts from M Bishop (UCSF) and T Yoshimori (Osaka University), respectively. The expression vector for CREB-ER was generated by ligating CREB CDNA and the ligand-binding domain of estrogen receptor $\alpha$ (ER) CDNA into pME18S. The expression vectors for M1CREB (CREB-S133A)-ER and KCREB (CREB-R301L)-ER were generated with a QuikChange Site-Directed Mutagenesis Kit (Stratagene). The expression vector for H2B-ECFP was generated by ligating $\mathrm{H} 2 \mathrm{~B}$ cDNA and ECFP cDNA amplified by PCR from a RIKEN FANTOM mouse cDNA clone (GenBank accession number AK018765) and pECFP-C1 (BD Biosciences), respectively, into pME18S-HygB (pME18S-Hyg-H2B-ECFP). Cells were transfected with expression vectors by using LipofectAMINE Plus (Invitrogen) according to the manufacturer's instructions. To obtain $\mathrm{CHO}$ cells stably expressing CREB-ER, cells were transfected with an expression vector of CREB-ER inserted into pME18S-neo. Two days after the transfection, cells were incubated with $0.7 \mu \mathrm{g} /$ ml G418 (Nacalai Tesque Inc.) for a week. A clone stably expressing CREB-ER was isolated and designated C32. To obtain C32 cells stably expressing H2B-ECFP and EYFP- $\alpha$-tubulin, cells were transfected with pME18S-Hyg-H2B-ECFP and pEYFPTub (BD biosciences). Two days after the transfection, cells were incubated with $0.7 \mu \mathrm{g} / \mathrm{ml}$ hygromycin B (Invitrogen) for a week. A clone stably expressing both H2B-ECFP and EYFP- $\alpha$-tubulin was selected under a fluorescence microscope.

Preparation of lentiviral vectors. Lentiviral vectors, provided by $\mathrm{H}$ Miyoshi (RIKEN), were prepared as described earlier. ${ }^{37}$ For the expression of CREB-ER, M1CREB-ER, or KCREB-ER, their CDNA fragments were inserted into CSII-SR $\alpha$ MCS-IRES-puro. For the expression of eEF1A1 or eEF1A2, a CDNA fragment of eEF1A1 cloned from pAN7 or that of eEF1A2 from the Mammalian Gene Collection (MGC) clone (clone number 8362) was inserted into CSII-SR $\alpha$-MCS-IRES-puro. For the expression of destabilized EGFP (d2EGFP), a cDNA fragment of d2EGFP cloned from pd2EGFP enhancer (BD Biosciences) was inserted into CSII-EF-MCSIRES-puro. For the expression of ECFP-Dcp1b, ECFP cDNA and Dcp1b cDNA were amplified by PCR from PECFP-C1 (BD Biosciences) and MGC clone (clone number 10639), respectively, and ligated into CSII-CMV-MCS. For gene silencing with the RNAi system, we used a lentivirus-based shRNA expression vector, CSIIU6 (provided by Y Satou and M Matsuoka, Kyoto University) and CSII-U6-puro vectors. The target sequences of the shRNAs are listed in Supplementary Table S1, and the shRNA-encoding DNA oligonucleotide insert sequences were generated by using Insert Design Tool for the pSilencer Vectors (Applied Biosystems, http:// www.ambion.com/techlib/misc/psilencer_converter.html).

Tetracycline-inducible shRNA expression system. To obtain the tetracycline-inducible shRNA expression vector (CSII-U6tet), the sequence between the TATA box and the transcription initiation site of the mouse U6 promoter of CSII-U6 ( $5^{\prime}$-TCCCTTGGAGAAAAGCCTT-3') was replaced with the sequence of the tetracycline operator (tetO) $\left(5^{\prime}\right.$-TCCCTATCAGTGATAGAGA-3'). The PGK promoter-driven neomycin phosphotransferase $\left(n e o^{r}\right)$ cDNA from pPGKneobpA was inserted into the Xhol/Xbal site of CSII-U6tet and the vector was named CSII-U6tet-neo. For the expression of the tetracycline repressor (TetR), a cDNA fragment of TetR from pcDNA6/TR (invitrogen) was inserted into CSII-EFMCS-IRES-puro. To obtain TetR-expressing cells, cells were infected with lentiviruses expressing TetR (CSII-EF-TetR-IRES-puro). After 2 days, cells were incubated with $10 \mu \mathrm{g} / \mathrm{ml}$ puromycin (Nacalai Tesque Inc.) and puromycin-resistant cells were amplified. The TetR-expressing cells were infected with lentiviruses expressing shRNA. After 2 days, cells were incubated with $0.7 \mu \mathrm{g} / \mathrm{ml} \mathrm{G418}$ for a week.

Quantification of cell death. To quantify cell death, the integrity of the cell membrane was measured by quantifying the amount of lactate dehydrogenase (LDH) released from dead cells using the CytoTox-ONE Homogeneous Membrane Integrity Assay (Promega). Cell death $(\%)=$ (amount of LDH released in culture medium)/(amount of LDH released in culture medium plus that retained in viable attached cells) $\times 100$. Caspase-independent cell death was analyzed by culturing cells in the presence of z-VAD-fmk. $z$-VAD-fmk was added every $24 \mathrm{~h}$ to sustain its inhibitory effect on caspase activity.

Flow cytometric analysis. For analyses of DNA content (cell cycle analysis), cells were labeled for 30 min with $10 \mu \mathrm{M}$ 5-bromo-2'-deoxy-uridine (BrdU) and fixed in $70 \%$ ethanol in $50 \mathrm{mM}$ glycine buffer $(\mathrm{pH} 2.0)$ for $30 \mathrm{~min}$ at $4^{\circ} \mathrm{C}$. After being washed with PBS, fixed cells were treated with $4 \mathrm{~N} \mathrm{HCl}$ for $20 \mathrm{~min}$. After another 
wash with PBS, cells were incubated in incubation buffer $(0.5 \%$ bovine serum albumin, $0.1 \%$ Tween 20 in PBS) for $10 \mathrm{~min}$, and stained with FITC-conjugated antiBrdU antibody (BD Pharmingen) diluted in the incubation buffer for $45 \mathrm{~min}$ at $37^{\circ} \mathrm{C}$. After further washing with PBS, cells were incubated with $10 \mu \mathrm{g} / \mathrm{ml} \mathrm{PI}$. For detection of cells with cleaved caspase-3, cells were stained using FITC-conjugated Monoclonal Active Caspase-3 Antibody Apoptosis Kit I (BD Pharmingen). For the TUNEL analysis, cells were stained using an in situ Cell Death Detection Kit, Fluorescein (Roche). In all the flow cytometric analyses, cells were analyzed with an EPICS XL Flow Cytometer (Beckman Coulter).

Metaphase chromosome spreads. For the observation of metaphase spreads of chromosomes, C32 cells were treated with $100 \mathrm{nM} \mathrm{4-OHT}$ for $2 \mathrm{~h}$, and then cultured for $6 \mathrm{~h}$ in the presence of $0.1 \mu \mathrm{g} / \mathrm{ml}$ colcemid and 4-OHT. After being washed with PBS, cells were incubated with $0.03 \mathrm{M}$ sodium citrate buffer, and fixed with methanol/acetic acid $(3: 1)$. Metaphase spreads were prepared as described earlier. $^{38}$

Time lapse imaging. For time lapse imaging, the C32-derived cell line stably expressing H2B-ECFP and EYFP- $\alpha$-tubulin was cultured in a Poly-L-lysine-coated glass bottom dish. After treatment with $100 \mathrm{nM} 4-\mathrm{OHT}$ for $12 \mathrm{~h}$, the dish was placed on a temperature-controlled stage maintained at $37^{\circ} \mathrm{C}$ in $5 \% \mathrm{CO}_{2}$. Time lapse imaging was performed on an inverted microscope (DMIRE2; Leica) with a $40 \times$ objective. ECFP and EYFP images were taken with a cooled CCD camera CoolSNAP HQ (Roper Scientific) driven by MetaMorph software (Molecular Devices).

Immunofluorescent staining. Cells in chamber slides were washed with PBS and fixed with $3.7 \%$ formaldehyde in PBS for 15 min. Fixed cells were permeabilized with $0.1 \%$ Triton X-100 in PBS for $15 \mathrm{~min}$, and blocked with $1 \%$ bovine serum albumin in PBS for $1 \mathrm{~h}$. Cells were treated with primary antibodies for $1 \mathrm{~h}$. After being washed with PBS, the cells were treated with Alexa Fluor 488 or Alexa Fluor 594-conjugated anti-mouse or rabbit lgG (1:1000; Molecular Probes) for $1 \mathrm{~h}$. To detect $F$-actin, the cells were treated with Texas Red-X phalloidin (Molecular Probes) together with the secondary antibodies. The cells were washed with PBS and mounted with VECTASHIELD Mounting Medium with DAPI (Vector Laboratories). The antibodies used in this study were those for $\mathrm{ER} \alpha(1: 100 ; \mathrm{HC}-20$; Santa Cruz), $\gamma$-tubulin (1:1000; GTU-88; Sigma), and eEF1A (1:100; CBP-KK1; Millipore)

cDNA subtraction. cDNA subtraction experiments were carried out using cDNA from C32 cells cultured with $100 \mathrm{nM} 4-\mathrm{OHT}$ in $\mathrm{EtOH}$ or EtOH only for $30 \mathrm{~h}$ using a PCR-Select cDNA Subtraction Kit (BD Biosciences) according to the manufacturer's instructions.

Northern blotting. Cellular total RNA was prepared from C32 cells treated with $100 \mathrm{nM} 4-\mathrm{OHT}$ for $30 \mathrm{~h}$ using ISOGEN (Nippon Gene) according to the manufacturer's instructions. Total RNA $(30 \mu \mathrm{g})$ was loaded on a formaldehydeagarose gel for electrophoresis and transferred to a nylon membrane. The membrane was hybridized with alkaline phosphate-labeled DNA probes for eEF1A1, elF5A, and $\beta$-actin. Probes were obtained by PCR using primers, sequences of which are listed in Supplementary Table S2. The labeling of probes and hybridization were carried out using AlkPhos Direct (GE Healthcare Bio-Sciences) according to the manufacturer's instructions.

Western blotting and antibodies. Cells were suspended in ice-cold lysis buffer ( $50 \mathrm{mM}$ Tris- $\mathrm{HCl}$ (pH 7.5), $150 \mathrm{mM} \mathrm{NaCl}, 5 \mathrm{mM}$ EDTA, $1 \%$ Triton X-100, and $0.5 \%$ sodium deoxycholate) containing a protease inhibitor cocktail (Nacalai Tesque Inc.). Cell lysates and immunoprecipitates were resolved by SDS-PAGE and analyzed by western blotting as described earlier. ${ }^{39}$ The antibodies used in this study were those for CREB (1:1000; Cell Signaling), caspase-3 (1:1000; Cell Signaling), cleaved caspase-3 (1:1000; Cell Signaling), PARP (1:1000; Cell Signaling), Lamin A/C (1:1000; Cell Signaling), eEF1A (1:5000; CBP-KK1 Millipore), actin (1:5000; C4; Chemicon), p53 (1:2000; 1C12; Cell Signaling), p21 (1:200; F-5; Santa Cruz), AIF (1:2000; E-1; Santa Cruz), cytochrome $c(1: 1000$ 7H8.2C12; BD Pharmingen), and Cox IV (1:5000; 20E8; Molecular Probes).

Real-time quantitative RT-PCR. Total RNA was isolated with a RNeasy Mini Kit (Qiagen) according to the manufacturer's instructions. Reverse transcription (RT) reactions were carried out with 8-20 ng of total RNA using a SuperScript III
Platinum SYBR Green One-Step qRT-PCR Kit (Invitrogen) according to the manufacturer's instructions. RT products were analyzed with an ABI PRISM 7500 (Applied Biosystems). Primer sequences are listed in Supplementary Table S3. The expression level of the mRNAs was normalized to that of $\beta$-actin, and expressed as a ratio to the expression level in untreated cells.

Fluorescence in situ hybridization. Fluorescence in situ hybridization (FISH) was performed as described earlier. ${ }^{40}$ The following oligonucleotide specific for eEF1A1 was used: $5^{\prime}$-GTTCT*GAGACCGTT ${ }^{*}$ CTTCCACCACT $^{*}$ GA TTAAGACT*GGGGTGGCAGGT*GTT-3'. Oligonuleotide was directly labeled with Alexa Flour 594 succinimidyl esters (Invitrogen) at amino-modified thymidine residues indicated by the asterisks.

RT-PCR. Total RNA was isolated with a RNeasy Mini Kit (Qiagen) according to the manufacturer's instructions. RT-PCR was carried out using a ThermoScript RT-PCR System (Invitrogen) according to the manufacturer's instructions. Primers specific for eEF1A1, eEF1A2 or $\beta$-actin are commonly used among human, mouse and Chinese hamster cells. Each primer sequence is definitely identical among human, mouse, and Chinese hamster, and is listed in Supplementary Table S4. To design the primers of eEF1A2, a partial sequence of Chinese hamster eEF1A2 gene was determined after cloning by PCR from genomic DNA of C32 cells using the following primer sets: forward, 5'-GTATGCCTGGGTGCTGGAC-3'; reverse, 5'GAGATGGGCACGAAGGGC-3'). The nucleotide sequence of Chinese hamster eEF1A2 that was obtained has been deposited in GenBank under the accession number FJ011555.

Acknowledgements. We thank J Fujisawa, T Yoshimori, M Bishop, $\mathrm{H}$ Miyoshi, Y Satou and M Matsuoka for vectors; and O Niwa for cells. We also thank T Iwano, Y Shinkai for methods; S Takeda and F Ishikawa for discussions; and all members of our laboratory for discussions and help. This study was supported by the Genome Network Project from the Ministry of Education, Culture, Sports, Science and Technology (MEXT) of Japan and Grants-in-Aid from the MEXT of Japan. YK was supported by the 21st Century Centers of Excellence (COE) Program of the MEXT Japan and the Research Fellowships of the Japan Society for the Promotion of Science for Young Scientists.

1. Yanagida M. Fission yeast cut mutations revisited: control of anaphase. Trends Cell Biol 1998; 8: 144-149.

2. Sakaguchi A, Kikuchi A. Functional compatibility between isoform alpha and beta of type II DNA topoisomerase. J Cell Sci 2004; 117 (Part 7): 1047-1054.

3. Ono T, Fang Y, Spector DL, Hirano T. Spatial and temporal regulation of condensins I and II in mitotic chromosome assembly in human cells. Mol Biol Cell 2004; 15: 3296-3298.

4. Ishida R, Miki T, Narita T, Yui R, Sato M, Utsumi KR et al. Inhibition of intracellular topoisomerase II by antitumor bis(2,6-dioxopiperazine) derivatives: mode of cell growth inhibition distinct from that of cleavable complex-forming type inhibitors. Cancer Res 1991; 51: 4909-4916.

5. Storchova Z, Pellman D. From polyploidy to aneuploidy, genome instability and cancer. Nat Rev Mol Cell Biol 2004; 5: 45-54.

6. Shi Q, King RW. Chromosome nondisjunction yields tetraploid rather than aneuploid cells in human cell lines. Nature 2005; 437: 1038-1042.

7. Fujiwara $T$, Bandi M, Nitta M, Ivanova EV, Bronson RT, Pellman D. Cytokinesis failure generating tetraploids promotes tumorigenesis in p53-null cells. Nature 2005; 437: 1043-1047.

8. Margolis RL, Lohez OD, Andreassen PR. G1 tetraploidy checkpoint and the suppression of tumorigenesis. J Cell Biochem 2003; 88: 673-683.

9. Uetake $Y$, Sluder $G$. Cell cycle progression after cleavage failure: mammalian somatic cells do not possess a 'tetraploidy checkpoint'. J Cell Biol 2004; 165: 609-615.

10. Wong $C$, Stearns T. Mammalian cells lack checkpoints for tetraploidy, aberrant centrosome number, and cytokinesis failure. BMC Cell Biol 2005; 6: 6 .

11. Zhivotovsky B, Kroemer G. Apoptosis and genomic instability. Nat Rev Mol Cell Biol 2004; 5: $752-762$.

12. Chipuk JE, Green DR. Do inducers of apoptosis trigger caspase-independent cell death? Nat Rev Mol Cell Biol 2005; 6: 268-275.

13. Okada H, Mak TW. Pathways of apoptotic and non-apoptotic death in tumour cells. Nat Rev Cancer 2004; 4: 592-603.

14. Feil R, Wagner J, Metzger D, Chambon P. Regulation of Cre recombinase activity by mutated estrogen receptor ligand-binding domains. Biochem Biophys Res Commun 1997; 237: 752-757

15. Gonzalez GA, Montminy MR. Cyclic AMP stimulates somatostatin gene transcription by phosphorylation of CREB at serine 133. Cell 1989; 59: 675-680. 
16. Walton KM, Rehfuss RP, Chrivia JC, Lochner JE, Goodman RH. A dominant repressor of cyclic adenosine $3^{\prime}, 5^{\prime}$-monophosphate (cAMP)-regulated enhancer-binding protein activity inhibits the CAMP-mediated induction of the somatostatin promoter in vivo. Mol Endocrinol 1992; 6: 647-655.

17. Mullins JM, Biesele JJ. Terminal phase of cytokinesis in D-98s cells. J Cell Biol 1977; 73 672-684

18. Norden C, Mendoza M, Dobbelaere J, Kotwaliwale CV, Biggins S, Barral Y. The NoCut pathway links completion of cytokinesis to spindle midzone function to prevent chromosome breakage. Cell 2006; 125: 85-98.

19. Susin SA, Lorenzo HK, Zamzami N, Marzo I, Snow BE, Brothers GM et al. Molecular characterization of mitochondrial apoptosis-inducing factor. Nature 1999; 397: 441-446.

20. Shimizu S, Kanaseki T, Mizushima N, Mizuta T, Arakawa-Kobayashi S, Thompson CB et al. Role of Bcl-2 family proteins in a non-apoptotic programmed cell death dependent on autophagy genes. Nat Cell Biol 2004; 6: 1221-1228.

21. Downes CS, Clarke DJ, Mullinger AM, Gimenez-Abian JF, Creighton AM, Johnson RT. A topoisomerase II-dependent G2 cycle checkpoint in mammalian cells. Nature 1994; 372 : 467-470.

22. Deming PB, Cistulli CA, Zhao H, Graves PR, Piwnica-Worms H, Paules RS et al. The human decatenation checkpoint. Proc Natl Acad Sci USA 2001; 98: 12044-12049.

23. Nakagawa T, Hayashita $Y$, Maeno K, Masuda A, Sugito N, Osada H et al. Identification of decatenation G2 checkpoint impairment independently of DNA damage G2 checkpoint in human lung cancer cell lines. Cancer Res 2004; 64: 4826-4832.

24. Cougot N, Babajko S, Seraphin B. Cytoplasmic foci are sites of mRNA decay in human cells. J Cell Biol 2004; 165: 31-40.

25. Parker R, Sheth U. P bodies and the control of mRNA translation and degradation. Mol Cell 2007; 25: 635-646.

26. Maser RS, DePinho RA. Connecting chromosomes, crisis, and cancer. Science 2002; 297 565-569.

27. Lee H, Larner JM, Hamlin JL. Cloning and characterization of Chinese hamster p53 cDNA. Gene 1997; 184: 177-183.

28. Zhu J, Hayakawa A, Kakegawa T, Kaspar RL. Binding of the La autoantigen to the $5^{\prime}$ untranslated region of a chimeric human translation elongation factor $1 \mathrm{~A}$ reporter mRNA inhibits translation in vitro. Biochim Biophys Acta 2001; 1521: 19-29.
29. Kahns S, Lund A, Kristensen P, Knudsen CR, Clark BF, Cavallius J et al. The elongation factor $1 \mathrm{~A}-2$ isoform from rabbit: cloning of the $\mathrm{CDNA}$ and characterization of the protein Nucleic Acids Res 1998; 26: 1884-1890.

30. Hornstein E, Tang $H$, Meyuhas $O$. Mitogenic and nutritional signals are transduced into translational efficiency of TOP mRNAs. Cold Spring Harb Symp Quant Biol 2001; 66 477-484.

31. Meyuhas 0 . Synthesis of the translational apparatus is regulated at the translational level. Eur J Biochem 2000; 267: 6321-6330.

32. Pellizzoni L, Lotti $F$, Rutjes $S A$, Pierandrei-Amaldi $P$. Involvement of the Xenopus laevis Ro60 autoantigen in the alternative interaction of $\mathrm{La}$ and CNBP proteins with the $5^{\prime}$ UTR of L4 ribosomal protein mRNA. J Mol Biol 1998; 281 593-608.

33. Lee S, Francoeur AM, Liu S, Wang E. Tissue-specific expression in mammalian brain heart, and muscle of $S 1$, a member of the elongation factor-1 alpha gene family. $J$ Bio Chem 1992; 267: 24064-24068.

34. Anand N, Murthy S, Amann G, Wernick M, Porter LA, Cukier IH et al. Protein elongation factor EEF1A2 is a putative oncogene in ovarian cancer. Nat Genet 2002; 31 301-305.

35. Tomlinson VA, Newbery HJ, Wray NR, Jackson J, Larionov A, Miller WR et al. Translation elongation factor eEF1A2 is a potential oncoprotein that is overexpressed in two-thirds of breast tumours. BMC Cancer 2005; 5: 113.

36. Kabeya Y, Mizushima N, Ueno T, Yamamoto A, Kirisako T, Noda T et al. LC3, mammalian homologue of yeast Apg8p, is localized in autophagosome membranes after processing. EMBO J 2000; 19: 5720-5728.

37. Okamoto K, Fujisawa J, Reth M, Yonehara S. Human T-cell leukemia virus typeoncoprotein tax inhibits Fas-mediated apoptosis by inducing cellular FLIP through activation of NF-kappaB. Genes Cells 2006; 11: 177-191.

38. Iwano T, Tachibana M, Reth M, Shinkai Y. Importance of TRF1 for functional telomere structure. J Biol Chem 2003; 279: 1442-1448.

39. Lee KK, Yonehara S. Phosphorylation and dimerization regulate nucleocytoplasmic shuttling of mammalian STE20-like kinase (MST). J Biol Chem 2002; 277: 12351-12358.

40. Chartrand P, Bertrand E, Singer RH, Long RM. Sensitive and high-resolution detection of RNA in situ. Methods in enzymology 2000; 318: 493-506.

\section{Supplementary Information accompanies the paper on Cell Death and Differentiation website (http://www.nature.com/cdd)}

